\title{
Energy Efficient Sensor, Relay and Base Station Placements for Coverage, Connectivity and Routing
}

\author{
Maulin Patel*, R. Chandrasekaran $\dagger$ and S.Venkatesan $\dagger$ \\ Telecommunication Engineering Program \\ Erik Jonsson School of Engineering and Computer Science \\ University of Texas at Dallas, Richardson, Texas 75083-0688 \\ Email:*maulin@student.utdallas.edu, †\{chandra, venky\}@utdallas.edu
}

\begin{abstract}
We consider a wireless sensor network made of sensor nodes capable of sensing and communication, relay nodes capable of communication, and base stations responsible for collecting data generated by sensor nodes, to be deployed in sensor field. We address the problem of placing the sensor nodes, relay nodes and base stations in the sensor field such that (i) each point of interest in the sensor field is covered by a subset of sensors of desired cardinality (ii) the resulting sensor network is connected and (iii) the sensor network has sufficient bandwidth. We propose several deployment strategies to determine optimal placements of sensor nodes, relay nodes and base stations for guaranteed coverage, connectivity, bandwidth and robustness. We study several different objectives such as minimizing the number of sensor nodes deployed, minimizing the total cost, minimizing the energy consumption, maximizing the network lifetime and maximizing the network utilization. The placement problems for reliable as well as unreliable/probabilistic detection models are formulated as Integer Linear Programs (ILPs). The practicality, effectiveness and performance of the proposed strategies are illustrated through simulations.
\end{abstract}

\section{INTRODUCTION}

Distributed sensor networks, designed to monitor and/or control the surrounding environmental phenomena, have the potential to revolutionize many applications. A sensor network consists of sensor nodes and one or more base stations. Sensor nodes generate, process, and forward data (via intermediate sensor nodes) to base stations. Among the major design challenges in the design of sensor networks is the efficient utilization of resources available to sensor nodes such as scarce bandwidth and limited energy supply. In applications such as surveillance, target tracking and intrusion detection, the strategic placement of sensor nodes in the sensor field can greatly enhance the sensing quality, reduce the cost and minimize the energy consumption, thereby increasing lifetimes of sensor nodes.

Depending on properties of sensing devices, terrain and application scenarios, the sensing capabilities of sensor nodes can be modeled as reliable (precise) or unreliable (imprecise). In the unreliable sensing model, a sensing/detection probability is assigned to each reading which characterizes the level of confidence in the reading. In the reliable detection model readings within the sensing range are assumed to be precise.

The area in which a sensor can perform its sensing, monitoring, surveillance and detection tasks with a reasonable accuracy (i.e. the sensor readings have at least a threshold level of sensing/detection probabilities within that area) is known as the coverage area. The union of coverage areas of individual sensors is the coverage area of a sensor network. Coverage areas can be irregular and can be location dependent due to the obstructions in the terrain e.g. sensors deployed for indoor applications, in urban and hilly areas [1]. The coverage area may also depend on the target, e.g. a sensor might be able to detect smaller targets from short distances and bigger targets from long distances.

The degree of the sensing coverage required depends on the application [2]. Covering every location with multiple sensors can provide robustness. Some applications may require the preferential coverage of critical locations based on risk or tactical importance [1]. The coverage requirements can also change with time due to changes in environmental conditions, e.g. the visibility can vary due to fog or smoke. A low degree of coverage might be sufficient in normal circumstances but, when a critical event is sensed, a high degree of coverage may be desired in that area [2].

It is desirable to achieve the required degree of coverage and robustness with the minimum number of active sensor nodes to minimize cost, interference and information redundancy [2], [3]. However, due to the limited range of the wireless communication and obstacles such as buildings, walls and trees, the minimum number of sensor nodes required for the coverage may not guarantee the connectivity of the resulting sensor network. Some technologies require the transmitter and the receiver to be in the line-of-sight, e.g. Infra-red, Ultrasound [4]. Therefore, a sensor node placement strategy must take connectivity and wireless channel bandwidth limitation (communication bottlenecks) into consideration when deciding on where to place sensor nodes.

Since the sensor nodes have limited energy supplies, a sensor node placement strategy, which minimizes the energy consumed in communication or maximizes the time till the required degree of coverage is maintained, is highly desirable.

In this paper, we propose several deployment strategies which determine the optimal placements of sensor nodes, relay nodes and base stations for guaranteed coverage, connectivity, bandwidth and robustness. The placement problems for reliable as well as probabilistic detection models are formulated as ILPs. The proposed strategies are compared using simulations. 


\section{RELATED WORK}

Sensor placement problem is closely related to the art gallery problem [5]. Chakrabarty et al. [6] have formulated the sensor placement problem as an ILP problem. Ray et al. [4] and Chakrabarty et al. [6] have used a framework of identifying codes to determine sensor placements. Zou and Chakrabarty [7] have proposed a virtual force algorithm to redeploy sensors to new position after initial random placement. Dasgupta et al. [8] have developed a Sensor Placement and Role assignment for energy-efficient INformation Gathering (SPRING) algorithm. Dhillon and Chakrabarty [1] have proposed algorithms for coverage optimization under the constraints of imprecise detections and terrain properties. Gandham et al. [9] have formulated the base station location problem as an ILP. Cheng et al. [10] have studied the impact of placing relays on the topology of biomedical sensor networks. Hou et al. [11] have formulated the problem of energy provisioning and relay placement into a mixed-integer non-linear programming problem.

\section{SYSTEM MODEL}

The following assumptions are made about the system.

- A sensor network consists of sensors $^{1}$, relays ${ }^{1}$ and base stations, all of these are static.

- Sensors are responsible for sensing/monitoring surrounding environment as well as forwarding data they receive from other sensors and relays towards base stations. Sensors generate data packets periodically and all packets are of the same size. Data aggregation is not performed.

- Relays are responsible for forwarding data they receive (from other sensors and relays) towards the base station.

- Base stations are responsible for collecting all the data generated by sensors. Base stations have sufficient hardware, sufficient software and constant power supply. All the base stations are homogeneous. Thus, a sensor can send data packets to any base station.

- The critical points are locations in the sensor field which must be covered by the required number of sensors.

- The feasible sites are the locations where it is possible to deploy sensors, relays or base stations.

- Sensors, relays and base stations can communicate with other nodes within their radio transmission range using a MAC protocol. The MAC protocol determines channel capacity i.e. the mean rate at which sensors and relays can transmit data to their neighbors over wireless channels.

- The predominant traffic in the network is the data traffic from sensors to the base stations.

The following notations are used in problem formulations.

$\Psi \quad$ Set of critical points in the sensor field.

$\Phi_{s} \quad$ Set of feasible sites for sensors and relays.

$\Phi_{b} \quad$ Set of feasible sites for base stations.

$\Phi \quad \Phi_{b} \cup \Phi_{s}$.

$R L_{\max }$ Upper bound on the number of relays that can be deployed.

\footnotetext{
${ }^{1}$ The word node is omitted to save the space.
}

$B_{\max }$ Upper bound on the number of base stations that can be deployed.

$\delta_{i m}= \begin{cases}1 & \begin{array}{l}\text { if critical point } m \in \Psi \text { is within the coverage } \\ \text { area of a sensor placed at location } i \in \Phi_{s}\end{array} \\ 0 & \text { otherwise. }\end{cases}$

$Q_{m} \quad$ Critical point $m \in \Psi$ must be covered by at least $Q_{m} \geq 1$ sensors.

$\Omega_{i} \subseteq \Phi$ Set of those feasible sites (neighbor set) which can be directly reached by a sensor/relay/base station placed at location $i \in \Phi$ with a certain power level .

$A \quad$ Set of all directed links $(i, j)$ where $i, j \in \Phi$ and $j \in \Omega_{i}$.

$N_{\max }$ Maximum number of sensors/relays that can be deployed within the wireless communication range of a sensor/relay excluding itself.

$D_{i} \quad$ The rate at which the information is generated at a sensor located at $i \in \Phi_{s}$.

$E \quad$ Energy supply available at a sensor/relay.

$G \quad$ Energy consumed in generating one unit of information at a sensor.

$T_{i j} \quad$ The energy required to transmit one unit of data over the link $(i, j) \in A$. Since base stations have constant power supply $T_{i j}=0$ if $i \in \Phi_{b}$.

$R_{j i} \quad$ The energy required to receive one unit of data at a sensor/relay/base station located at $j \in \Phi . R_{j i}=0$ if $j \in \Phi_{b}$.

$U_{i j} \quad$ Capacity of the wireless link $(i, j) \in A$.

$U_{\max }$ Maximum amount of data a sensor/relay can handle (transmit and/or receive) per unit time (called the node capacity).

$x_{i}= \begin{cases}1 & \text { if a sensor is placed at location } i \in \Phi_{s} \\ 0 & \text { otherwise. }\end{cases}$

$y_{i}= \begin{cases}1 & \text { if a relay is placed at location } i \in \Phi_{s} \\ 0 & \text { otherwise. }\end{cases}$

$z_{k}= \begin{cases}1 & \text { if a base station is placed at location } k \in \Phi_{b} \\ 0 & \text { otherwise. }\end{cases}$

$f_{i j} \quad$ The data flow rate over the wireless link $(i, j) \in A$.

$l>0$ Lifetime of the network (i.e. time till the required degree of coverage is maintained).

In the following sections we assume that sensor readings are reliable. In section IX we extend our ILP formulations for probabilistic sensing model.

\section{Minimum Sensor Placement (MSP)}

Given a set of critical points to be covered, a set of feasible sites for the sensor placement, sensing areas of sensors to be placed at feasible sites, parameter $Q_{m} \geq 1$, determine the minimum number of sensors and their placements at the feasible sites such that each critical point $m \in \Psi$ is covered by at least $Q_{m}$ sensors.

$$
\operatorname{Obj}=\operatorname{Min} \sum_{\left\{i: i \in \Phi_{s}\right\}} x_{i}
$$

Subject to

$$
\sum_{\left\{i: i \in \Phi_{s}\right\}} \delta_{i m} \cdot x_{i} \geq Q_{m} \quad \forall m \in \Psi
$$

$$
x_{i} \in\{0,1\}, i \in \Phi_{s} .
$$


If $Q_{m}=1 \forall m \in \Psi$ then the above problem is an instance of the set cover problem. The set cover problem is a well known NP-complete problem for sub-sets of size 3 or more.

\section{Minimum Cost Placement (MCP)}

The objective is to achieve desired degree of coverage at the minimum total cost while being able to deliver all the data packets generated by sensors to base stations without exceeding the link and the node capacities. Let $\alpha, \beta, \gamma$ denote the cost of a sensor, a relay and a base station respectively. Formally, the problem can be stated as follows.

$$
O b j=\operatorname{Min} \sum_{\left\{i: i \in \Phi_{s}\right\}}\left(\alpha \cdot x_{i}+\beta \cdot y_{i}\right)+\sum_{\left\{k: k \in \Phi_{b}\right\}} \gamma \cdot z_{k}
$$

Subject to

$$
\begin{aligned}
& \sum_{\{j:(i, j) \in A\}} f_{i j}-\sum_{\{j:(j, i) \in A\}} f_{j i}=D_{i} \cdot x_{i} \quad \forall i \in \Phi_{s}, \\
& \sum_{\left\{k: k \in \Phi_{b}\right\}}\left(\sum_{\{j:(k, j) \in A\}} f_{k j}-\sum_{\{j:(j, k) \in A\}} f_{j k}\right)=-\sum_{\left\{i: i \in \Phi_{s}\right\}} D_{i} \cdot x_{i}, \\
& \sum_{\{j:(i, j) \in A\}} f_{i j}+\sum_{\{j:(j, i) \in A\}} f_{j i} \leq U_{\max } \cdot x_{i}+U_{\max } \cdot y_{i} \quad \forall i \in \Phi_{s}, \quad \text { (2c) } \\
& \sum_{\left\{i: i \in \Phi_{s}\right\}} \delta_{i m} \cdot x_{i} \geq Q_{m} \quad \forall m \in \Psi \\
& \sum_{\left\{k: k \in \Phi_{b}\right\}} z_{k} \leq B_{\max }, \\
& x_{i}+y_{i} \leq 1 \quad \forall i \in \Phi_{s}, \\
& x_{i}, y_{i}, z_{k} \in\{0,1\}, i \in \Phi_{s}, k \in \Phi_{b}, \\
& 0 \leq f_{i j} \leq U_{i j} \cdot x_{i}+U_{i j} \cdot y_{i} \quad \forall i \in \Phi_{s},(i, j) \in A, \\
& 0 \leq f_{i j} \leq U_{i j} \cdot x_{j}+U_{i j} \cdot y_{j} \quad \forall j \in \Phi_{s},(i, j) \in A, \\
& 0 \leq f_{i j} \leq U_{i j} \cdot z_{i} \quad \forall i \in \Phi_{b},(i, j) \in A, \\
& 0 \leq f_{i j} \leq U_{i j} \cdot z_{j} \quad \forall j \in \Phi_{b},(i, j) \in A .
\end{aligned}
$$

The first set of constraints (2a) ensures flow conservation at each sensor and relay. The second constraint $(2 b)$ ensures that the base stations receive all the data generated by sensor nodes. The sum of incoming and outgoing flow through a senor/relay should not exceed its capacity and this is ensured by the third set of constraints (2c). The fourth set of constraints (2d) ensures that each critical point $m \in \Psi$ is covered by at least $Q_{m} \geq 1$ sensors. The fifth constraint (2e) limits the maximum number of base stations that can be deployed. The sixth set of constraints (2f) ensures that a sensor and a relay, both are not deployed at the same feasible site. Note that this condition also means $\sum_{\left\{i: i \in \Phi_{s}\right\}} x_{i}+\sum_{\left\{i: i \in \Phi_{s}\right\}} y_{i} \leq\left|\Phi_{s}\right|$. The binary variables $(2 \mathrm{~g})$ determine the placement of sensors, relays and base stations. The remaining sets of constraints (2h), (2i), (2j), (2k) ${ }^{2}$ ensure that the flow on a wireless link is nonnegative and the flow does not exceed link's capacity. Note that the flow on a link connecting feasible sites can be positive only when sensor(s)/relay(s)/base station(s) are deployed at both (transmitting and receiving) ends of the link.

\footnotetext{
${ }^{2}$ Some of these constraints are mentioned for clarity.
}

\section{Vi. Minimum Energy Placement (MEP)}

Energy efficiency is a primary concern in the design of a wireless sensor network. Since a significant amount of energy is consumed in transmitting high volume of data generated by sensors, one objective is to minimize total energy consumed in communication.

$$
O b j=\operatorname{Min} \sum_{(i, j) \in A}\left(T_{i j}+R_{j i}\right) \cdot f_{i j}
$$

Subject to all the constraints of the MCP problem plus the following constraints.

$$
\begin{gathered}
\sum_{\left\{i: i \in \Phi_{s}\right\}} y_{i} \leq R L_{\max } \\
\sum_{\left\{j: j \in \Omega_{i}\right\}} x_{j}+\sum_{\left\{j: j \in \Omega_{i}\right\}} y_{j} \leq N_{\max }+\left|\Phi_{s}\right|-\left|\Phi_{s}\right| \cdot\left(x_{i}+y_{i}\right) \quad \forall i \in \Phi_{s} .
\end{gathered}
$$

The constraint in (3a) limits the number of relays that can be deployed. The interference caused by neighboring nodes can be reduced by limiting the number of neighbors a sensor/relay can have as shown in (3b). If desired, the base stations can be counted as neighbors by adding the term $\sum_{\left\{j: j \in \Omega_{i}\right\}} z_{j}$ on the left hand side of the set of constraints in (3b).

\section{MAXimum Lifetime Placement (MLP)}

In sensor networks the predominant traffic is the data traffic from sensors to base stations. The nodes close to the base stations forward significantly higher number of packets. Thus nodes close to base stations deplete their energies faster than the nodes that are far away. We define the network lifetime as the time till the required degree of coverage is maintained. It is desirable to place nodes such that the network lifetime is maximized.

$$
O b j=\operatorname{Max} \quad l
$$

Subject to

$$
\begin{gathered}
\sum_{\{j:(i, j) \in A\}} f_{i j} \cdot l-\sum_{\{j:(j, i) \in A\}} f_{j i} \cdot l=D_{i} \cdot l \cdot x_{i} \quad \forall i \in \Phi_{s} \\
\sum_{\left\{k: k \in \Phi_{b}\right\}}\left(\sum_{\{j:(k, j) \in A\}} f_{k j} \cdot l-\sum_{\{j:(j, k) \in A\}} f_{j k} \cdot l\right)=-\sum_{\left\{i: i \in \Phi_{s}\right\}} D_{i} \cdot l \cdot x_{i} \\
\sum_{\{j:(i, j) \in A\}} f_{i j} \cdot l+\sum_{\{j:(j, i) \in A\}} f_{j i} \cdot l \leq U_{\max } \cdot l \cdot x_{i}+U_{\max } \cdot l \cdot y_{i} \forall i \in \Phi_{s}, \quad \text { (4c) } \\
\sum_{\{j:(i, j) \in A\}} T_{i j} \cdot f_{i j} \cdot l+\sum_{\{j:(j, i) \in A\}} R_{j i} \cdot f_{j i} \cdot l+G \cdot D_{i} \cdot l \cdot x_{i} \leq E \cdot x_{i}+E \cdot y_{i} \forall i \in \Phi_{s} \\
\sum_{\left\{i: i \in \Phi_{s}\right\}} \delta_{i m} \cdot x_{i} \geq Q_{m} \\
\sum_{\left\{j: j \in \Omega_{i}\right\}} x_{j}+\sum_{\left\{j: j \in \Omega_{i}\right\}} \leq N_{\max }+\left|\Phi_{s}\right|-\left|\Phi_{s}\right| \cdot\left(x_{i}+y_{i}\right) \quad \forall i \in \Phi_{s}, \\
\sum_{\left\{k: k \in \Phi_{b}\right\}} z_{k} \leq B_{\max }, \\
\sum_{\left\{i: i \in \Phi_{s}\right\}} y_{i} \leq R L_{\max } \\
x_{i}+y_{i} \leq 1 \\
\forall i \in \Phi_{s}
\end{gathered}
$$




$$
\begin{array}{cc}
x_{i}, y_{i}, z_{k} \in\{0,1\}, i \in \Phi_{s}, k \in \Phi_{b}, \\
0 \leq f_{i j} \cdot l \leq U_{i j} \cdot l \cdot x_{i}+U_{i j} \cdot l \cdot y_{i} & \forall i \in \Phi_{s},(i, j) \in A, \\
0 \leq f_{i j} \cdot l \leq U_{i j} \cdot l \cdot x_{j}+U_{i j} \cdot l \cdot y_{j} & \forall j \in \Phi_{s},(i, j) \in A, \\
0 \leq f_{i j} \cdot l \leq U_{i j} \cdot l \cdot z_{i} & \forall i \in \Phi_{b},(i, j) \in A, \\
0 \leq f_{i j} \cdot l \leq U_{i j} \cdot l \cdot z_{j} & \forall j \in \Phi_{b},(i, j) \in A, \\
0<l . &
\end{array}
$$

$(4 \mathrm{j})$

The constraints in the optimization model are nonlinear because they involve products of variables. In order to linearize the constraints, we divide both sides of constraints involving variable $l$ by $l>0$. We introduce a new variable $\bar{l}$ to replace $1 / l$. This will linearize all the constraints except (4d). To linearize $(4 \mathrm{~d})$, we replace $E \cdot x_{i} \cdot \bar{l}+E \cdot y_{i} \cdot \bar{l}$ with $E \cdot \bar{l}$. This transformation is valid because at least one of the binary variables $x_{i}$ and $y_{i}$ must be 0 (see $4 \mathrm{i}$ ). When both $x_{i}$ and $y_{i}$ are 0 then set of constraints in $(4 \mathrm{~d})$ are equivalent to the set of constraints in (4k) and in (4l). Maximizing the lifetime (l) in the above problem is equivalent to minimizing $\bar{l}$ in the transformed ILP problem.

\section{Maximum Utilization Placement (MUP)}

In order to improve the lifetime of the network MLP places many nodes close to the base station(s). This may result in poor utilization of network resources and increased cost. It is highly desirable to maximize the network lifetime while deploying a reasonable number of nodes. The network utilization (NU) is defined as $\frac{l}{\sum_{\left\{i: i \in \Phi_{s}\right\}}\left(x_{i}+y_{i}\right)}$.

$$
O b j=\operatorname{Max} \frac{l}{\sum_{\left\{i: i \in \Phi_{s}\right\}}\left(x_{i}+y_{i}\right)}=\operatorname{Min} \frac{\sum_{\left\{i: i \in \Phi_{s}\right\}}\left(x_{i}+y_{i}\right)}{l}
$$

Subject to all the constraints of MLP problem.

In order to linearize constraints we divide both sides of constraints involving variable $l$ by $l>0$. We introduce a new variable $\bar{l}$ to replace $1 / l$. Now (5) involves product of a binary variable $\left(x_{i}\right)$ with a continuous variable $(\bar{l})$. It can be linearized using the results of [12] by (a) replacing the product term with a new continuous variable $v_{i}$, and (b) adding additional set of constraints (i) $v_{i} \leq \bar{L}_{\max } \cdot x_{i}$, (ii) $v_{i} \leq \bar{l}$ and (iii) $\bar{l}-v_{i}+\bar{L}_{\max } \cdot x_{i} \leq \bar{L}_{\max }$ where $\bar{L}_{\max }$ is an upper bound for $\bar{l}$. Similarly, linearize the product of binary variable $y_{i}$ with continuous variable $\bar{l}$.

\section{Placement PROBlem FOR UNRELIABLE/PROBABILISTIC DETECTION MODEL}

A probabilistic sensing/detection model is useful when the sensor readings are unreliable. Let $p_{i m}$ denote the probability that an event occurring at a critical point $m \in \Psi$ is sensed/detected by a sensor placed at the feasible site $i \in \Phi_{s}$. The goal is to place the minimum number of sensors in the sensor field such that an event occurring at any critical point $m \in \Psi$ is detected with a probability of at least $O_{m}$. Due to the preferential coverage requirements, different critical points can have different threshold detection probabilities.

$$
O b j=\operatorname{Min} \sum_{\left\{i: i \in \Phi_{s}\right\}} x_{i}
$$

Subject to

$$
\begin{gathered}
1-\prod_{\left\{i: i \in \Phi_{s}\right\}}\left(1-p_{i m} \cdot x_{i}\right) \geq O_{m} \quad \forall m \in \Psi, \\
x_{i} \in\{0,1\}, i \in \Phi_{s} .
\end{gathered}
$$

Clearly, the problem is non-linear because the first set of constraints in (6a) involves product of variables. Simplifying the constraint and taking $\log$ of both sides of the constraint will result into the following set of inequality constraint.

$$
\sum_{\left\{i: i \in \Phi_{s}\right\}} \log \left(1-p_{i m} \cdot x_{i}\right) \leq \log \left(1-O_{m}\right) \quad \forall m \in \Psi
$$

Note that if $x_{i}$ is zero then $\log \left(1-p_{i m} \cdot x_{i}\right)$ is zero and if $x_{i}$ is one then $\log \left(1-p_{i m} \cdot x_{i}\right)$ is $\log \left(1-p_{i m}\right)$. The above set of non-linear constraint can be converted into the following set of linear constraint.

$$
\sum_{\left\{i: i \in \Phi_{s}\right\}} \log \left(1-p_{i m}\right) \cdot x_{i} \leq \log \left(1-O_{m}\right) \quad \forall m \in \Psi
$$

The ILP formulations studied in the previous sections can be easily extended for a probabilistic detection model by transforming non-linear constraints into linear constraints as shown above.

\section{Communication Model}

In adaptive transmission power model, the transmitter is capable of adjusting its signal power level such that the energy consumed in transmission is minimized while maintaining acceptable signal to noise ratio at the receiver. In constant transmission power model, the transmitter transmits with a constant signal power.

The energy consumed in transmission includes the energy consumed in internal processing (distance independent) and the energy consumed in amplifying the signal to achieve acceptable signal to noise ratio at a receiver (distance dependent). In adaptive transmission power model used in simulations, energy consumed in transmitting a bit from node $i$ to $j$ is given by [13]

$$
T_{i j}=\left(100+0.1 \times \operatorname{Dist}(i, j)^{3}\right) \times 10^{-9} \quad \text { Energy_unit } / \text { bit }
$$

In the constant transmission power model used in simulations, the term $\operatorname{Dist}(i, j)^{3}$ in the above equation is replaced by a constant Transmission_Range ${ }^{3}$ where Transmission_Range is fix (static) maximum transmission range of a node.

The energy consumed in receiving/generating a bit is the same in both models which is given by

$$
R=G=100 \times 10^{-9} \quad \text { Energy_unit/bit }
$$

\section{Simulation Results}

A sensor field of size $100 \mathrm{~m} \times 100 \mathrm{~m}$ is divided into a uniform grid of $21 \times 21$. The grid points represent the critical points to be covered and the feasible sites for sensors as well as relays. Four feasible sites for base stations are located as 


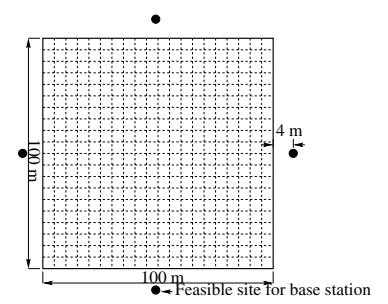

Fig. 1. Sensor Field

TABLE I

COMPARISON OF NUMBER OF SENSOR NODES REQUIRED

\begin{tabular}{|c|c|c|c|c|c|c|c|c|}
\hline$Q_{\min }$ & MSP & MCP & $\begin{array}{c}\text { MEP } \\
(\mathrm{A})\end{array}$ & $\begin{array}{c}\text { MEP } \\
(\mathrm{C})\end{array}$ & $\begin{array}{c}\text { MLP } \\
(\mathrm{A})\end{array}$ & $\begin{array}{c}\text { MLP } \\
(\mathrm{C})\end{array}$ & $\begin{array}{c}\text { MUP } \\
(\mathrm{A})\end{array}$ & $\begin{array}{c}\text { MUP } \\
(\mathrm{C})\end{array}$ \\
\hline 1 & 49 & 49 & 61 & 58 & 101 & 104 & 79 & 74 \\
\hline 2 & 105 & 105 & 116 & 112 & 166 & 168 & 131 & 168 \\
\hline 3 & 161 & 161 & 168 & 170 & 213 & 212 & 213 & 212 \\
\hline
\end{tabular}

shown in Fig.1. We have simulated a 2D sensor field with circular transmission and sensing areas under reliable sensing model. The transmission range of each sensor as well as relay is 20.0 meters. The sensing radius of each sensor is 8.0 meters. The node capacity of each sensor as well as relay is 40.0 $\mathrm{Kbps}^{3}$, and the link capacity of each wireless link is $10.0 \mathrm{Kbps}$. The rate of information generation is 512 bits/second for each sensor. The costs of sensors, relays and base stations are 10 units, 8 units and 500 units respectively. Each sensor/relay is equipped with initial energy of 100.0 Energy_unit. CPLEX ${ }^{\circledR}$ 8.1.0 optimizer [14] is used to solve ILP formulations. The character ' $\mathrm{C}$ ' inside the parenthesis (e.g. $\operatorname{MEP}(\mathrm{C})$ ) denotes that the formulation uses constant transmission power model and the character ' $A$ ' inside the parenthesis (e.g. MLP(A)) denotes that the formulation uses the adaptive transmission power model.

The first set of experiments (see Table I) compares the number of sensors required by each scheme for various coverage requirements $\left(Q_{\min }\right)$. To restrict the comparison to sensors, no relays are deployed $\left(R L_{\max }=0\right.$ and $\left.N_{\max }=\infty\right)$. The upper bound on number of base stations that can be deployed $\left(B_{\max }\right)$ is set to 4 and $Q_{m}=Q_{\min } \forall m \in \Psi$. Since the cost of a base station is significantly higher than the cost of a sensor, MCP solutions require only 1 base station. On the other hand MEP, MLP and MUP solutions require 4 base stations because deploying more base stations improves the objective function values. Note that the values of MLP and MUP solutions reported in Table I may not be optimal ${ }^{4}$.

Results in Table. I indicate that the minimum number of sensors required for coverage (MSP) increases linearly with $Q_{\min }$. Note that the graph induced by the wireless connectivity of the sensors (deployed by MSP) is connected and the resulting network has sufficient bandwidth. Therefore, the number of sensors required by MSP and MCP are the same.

In MEP, MLP and MUP, placing additional sensors (in addition to the minimum sensors required for the coverage,

\footnotetext{
${ }^{3}$ As per the IEEE 802.15.4 standard, the raw data rate of a sensor can be 20,40 or $250 \mathrm{~Kb} / \mathrm{s}$.

${ }^{4}$ The processes were terminated after $30 \mathrm{hrs}$ of computation.
}

connectivity and bandwidth) can save the energy and improve the objective function. However, additional sensors generate data which consume energy. In MEP, placing additional sensors is advantageous if the energy saved in transmission is more than energy consumed by additional data in reaching the base station.

The nodes close to base stations are overloaded with forwarding tasks and their lifetimes are significantly shorter than the lifetimes of other nodes. MLP and MUP attempt to improve the lifetimes of these nodes by placing many nodes close to base stations.

In MLP, additional sensors can be placed if the energy consumed by additional data does not decrease the network lifetime. Therefore, MLP formulations deploy more nodes than other formulations. In MUP, additional sensors can be placed if the increase in the network lifetime due to additional sensors is at least proportional to the number of additional sensors.

The next set of experiments compare NU of the networks synthesized in the previous experiments. In these experiments, given the sensor positions (determined by the first set of experiments in Table I) and base station positions (4 base stations are located as shown in Fig.1), the objective is to maximize the time till the desired coverage is maintained. Dividing the network lifetime by the number of nodes deployed gives NU.

Fig. 2 shows the NU of the networks under constant and adaptive transmission power models. The NU of the networks synthesized by MSP and MCP are the same because the sensor positions determined by MSP and MCP in the first set of experiments happen to be identical. Since MSP and MCP do not take energy efficiency into consideration, their $\mathrm{NU}$ values are the least.

Networks synthesized by MUP have the highest NU followed by the MLP. Clearly, a judicious placement of sensors in the sensor field can significantly increase the network lifetime.

The NU of networks in the adaptive transmission power models are roughly double the NU in constant transmission power models. Clearly, equipping sensors with power control transmitters can significantly increase the NU.

To study the effect of relay placements on objective function values, we iteratively solved the MEP problems (increasing the values of $R L_{\max }$ by 1 in each iteration) until the objective function values stop improving. MUP problems are solved by setting $R L_{\max }=\infty$. The number of sensor and relay nodes required are shown in Table II. The saving values show the percentage improvements in the objective function values due to the placement of relay nodes with respect to the objective function values when relay nodes are not deployed.

In the adaptive transmission power model the multi-hop communication is more energy efficient than direct transmission. However, at short distances, the energy consumed in internal processing and receiving the data dominates which makes direct transmission more efficient than multi-hop transmission. Therefore, as the node density increases (average distance between nodes decreases), the number of additional sensors and number of relays decrease (See Table. I and II). The saving percentage drops with the increase in node density. 


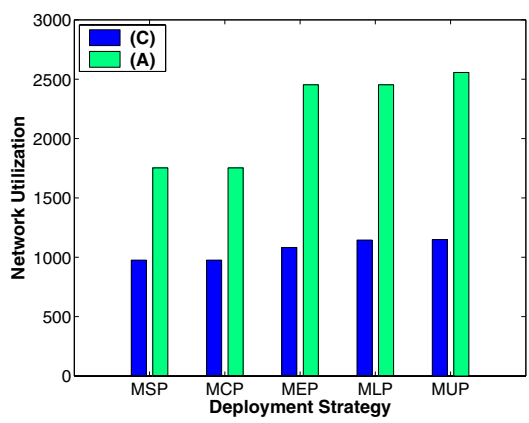

(a) $Q_{\min }=1$

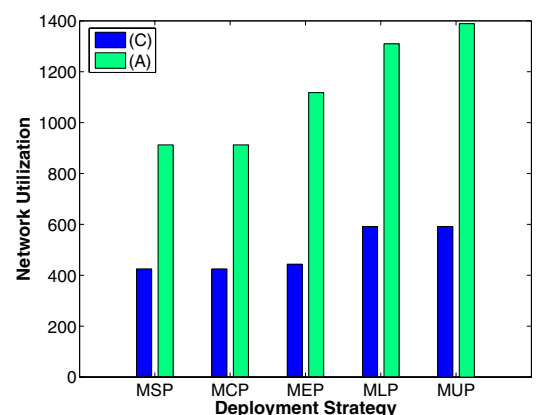

(b) $Q_{\min }=2$

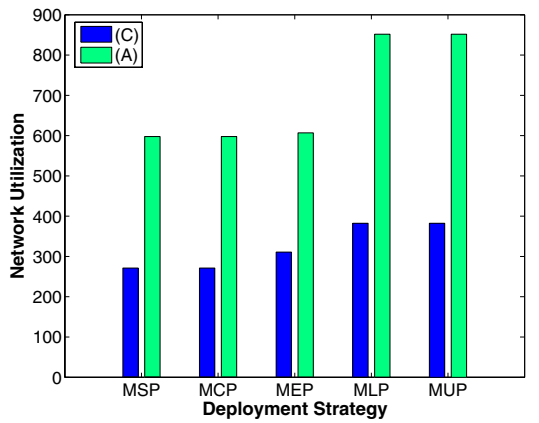

(c) $Q_{\min }=3$

Fig. 2. Deployment Strategies v/s Network Utilization

TABLE II

COMPARISON OF NUMBER OF RELAY NODES REQUIRED

\begin{tabular}{|c|c|c|c|c|c|c|c|c|c|c|c|c|}
\hline \multirow[t]{2}{*}{$Q_{\text {min }}$} & \multicolumn{3}{|c|}{ MEP(A) } & \multicolumn{3}{|c|}{$\mathrm{MEP}(\mathrm{C})$} & \multicolumn{3}{|c|}{ MUP(A) } & \multicolumn{3}{|c|}{ MUP(C) } \\
\hline & Sensors & Relays & Saving & Sensors & Relays & Saving & Sensors & Relays & Saving & Sensors & Relays & Saving \\
\hline 1 & 49 & 68 & $17.14 \%$ & 54 & 4 & $3.74 \%$ & 49 & 81 & $33.55 \%$ & 49 & 50 & $40.77 \%$ \\
\hline 2 & 105 & 85 & $8.56 \%$ & 110 & 4 & $0.92 \%$ & 105 & 73 & $29.25 \%$ & 108 & 76 & $35.96 \%$ \\
\hline
\end{tabular}

In the constant power model energy can be saved by minimizing the number of hops the data packets have to traverse to reach a base station. Since the minimum hop path can be abridged at relatively few places, the number of relays required and the percentage saving values are smaller in $\operatorname{MEP}(\mathrm{C})$ than in MEP(A). Results in Table. II indicate that relay placements improve the NU of networks synthesized by MUP significantly. Overall, MUP strategy is better than other deployment strategies.

\section{XiI. CONCLusion And Future Work}

In this paper, we investigated the joint problem of determining sensor, relay and base station placements, and finding bandwidth-constrained energy-efficient routes while ensuring desired level of coverage, connectivity and robustness. The practicality, effectiveness and performance of proposed strategies have been studied through simulations.

Our techniques of determining strategic placements of nodes are useful when a modest number of nodes are to be deployed and reasonable terrain information is available. For example, in many indoor applications (involving factories or industrial units or airports) where floor plans are available, our approaches are readily applicable.

We recognize that ILP formulations are NP-hard to solve. Since placement problems are off-line problems, more computational time and power can be devoted for their solutions. Hence, modest size of problems can be solved to optimality and good feasible solutions can be obtained for large size of problems using techniques such as LP Relaxation or Lagrangian Relaxation [15]. Moreover, the solutions of ILP formulations can be useful in benchmarking an approximation algorithm or a heuristic. It is interesting to develop polynomial time heuristic algorithms for large size of problems.

\section{REFERENCES}

[1] S. S. Dhillon and K. Chakrabarty, "Sensor placement for effective coverage and surveillance in distributed sensor networks," in Proceedings of IEEE WCNC, New Orleans, LA, March 2003, pp. 1609-1614.

[2] X. Wang, G. Xing, Y. Zhang, C. Lu, R. Pless, and C. Gill, "Integrated coverage and connectivity configuration in wireless sensor networks," in Proceedings of ACM SenSys 2003, Los Angeles, California, Nov. 2003, pp. 28-39.

[3] H. Zhang and J. C. Hou, "Maintaining sensing coverage and connectivity in large sensor networks," University of Illinois at Urbana-Champaign, IL, Tech. Rep. UIUCDCS-R-2003-2351, June 2003.

[4] S. Ray, R. Ungrangsi, F. D. Pellegrini, A. Trachtenberg, and D. Starobinski, "Robust location detection in emergency sensor networks," in Proceedings of IEEE INFOCOM '03, San Francisco, April 2003, pp. 1044-1053.

[5] J.O'Rourke, Art Gallery Theorems and Algorithms. Oxford University Press, New York, NY, 1987.

[6] K. Chakrabarty, S. S. Iyengar, H. Qi, and E. Cho, "Grid coverage for surveillance and target location in distributed sensor networks," IEEE Transactions on Computers, vol. 51, no. 12, pp. 1448-1453, Dec. 2002.

[7] Y. Zou and K. Chakrabarty, "Sensor deployment and target localization based on virtual forces," in Proceedings of IEEE INFOCOM '03, San Francisco, Apr. 2003, pp. 1293-1303.

[8] K. Dasgupta, M. Kukreja, and K. Kalpakis, "Topology-aware placement and role assignment for energy-efficient information gathering in sensor networks," in Proceedings of the 8th IEEE Symposium on Computers and Communications, Kemer-Antalya, Turkey, June 2003.

[9] S. R. Gandham, M. Dawande, R. Prakash, and S. Venkatesan, "Energy efficient schemes for wireless sensor networks with multiple mobile base stations," in Proceedings of IEEE GLOBECOM, San Francisco, CA, Dec. 2003.

[10] X. Cheng, D.-Z. Du, L. Wang, and B. Xu, "Relay sensor placement in wireless sensor networks," University of Minnesota Twin Cities, Minneapolis, MN, Tech. Rep. 02-004, 2001.

[11] Y. Hou, Y. Shi, and H. Sherali, "On energy provisioning and relaying node placement for wireless sensor networks," The Bradley Dept. of ECE, Virginia Tech, Blacksburg, VA, Tech. Rep., 2003.

[12] H. P. Williams, Model Building in Mathematical Programming. Chichester, England; New York: Wiley, 1999.

[13] W. Heinzelman, A. Chandrakasan, and H. Balakrishnan, "Energyefficient communication protocol for wireless micro-sensor networks," in Proc. of the 33rd HICSS, Maui, Hawaii, Jan. 2000, pp. 3005 - 3014.

[14] Using the CPLEX callable library, ILOG Inc., Incline Village, NV, 1997.

[15] R. Ahuja, T. Magnanti, and J. Orlin, Network Flows. New Jersey: Prentice Hall, 1993. 